\title{
Tecnura
}

\section{Desempeño de un material granular estabilizado con cemento cuando se aplica carga a compresión}

\section{Performance of a granular material stabilized with cement when compressive loading is applied}

Edgar Humberto Sánchez Cotte', Gleidys María Torres Chueco², Rodrigo Elías Esquivel Ramírez ${ }^{3}$

Fecha de recepción: 12 de septiembre de 2018

Fecha de aceptación: 14 de febrero de 2019

Cómo citar: Sánchez C., E.H, Torres C., GM. y Esquivel R., R.E. (2019). Desempeño de un material granular estabilizado con cemento cuando se aplica carga a compresión. Tecnura, 23(60), 59-71. DOI: https://doi. org/10.14483/22487638.14640

\section{Resumen}

Contexto: Esta investigación evalúa la resistencia a la compresión de un material granular mezclado compuesto por arcilla y arena, con el objetivo de optimizar su diseño.

Métodología: Los factores estudiados fueron el contenido de cemento, la humedad, los golpes de compactación y la relación arcilla/arena. Además, se realizó un diseño experimental inical de $2^{4}$.

Resultados: Los resultados permitieron identificar que el factor significativo es el contenido de cemento. Por otro lado, se exploró para la optimización un diseño de un solo factor con tres niveles $(175 ; 200$; y $225 \mathrm{Kg} / \mathrm{m}^{3}$ ) y dos réplicas.

Conclusiones: El diseño experimental permitió identificar el valor óptimo del factor contenido de cemento en la frontera de la región de experimentación seleccionada $\left(150 \mathrm{Kg} / \mathrm{m}^{3}\right)$.

Palabras clave: Sub base, estabilización, suelo cemento, ANOVA.

\begin{abstract}
Context: This research evaluates the compressive strength of a mixed granular material composed of clay and sand, in order to optimize its design.

Methodology: The factors studied were the cement content, the humidity, the compaction blows, and the clay/sand ratio. Additionally, an initial experimental design of $2^{4}$ was performed.

Results: The results allowed to identify that the significant factor is the cement content. On the other hand, a single-factor design with three levels (175; 200; and $225 \mathrm{Kg} / \mathrm{m}^{3}$ ) and two replicas was explored for optimization.
\end{abstract}

Conclusions: The experimental design allowed to identify the optimal value of the cement content factor in the border of the selected experimental region $\left(150 \mathrm{Kg} / \mathrm{m}^{3}\right)$.

Keywords: Sub base, stabilization, cement floor, ANOVA.

\footnotetext{
Ingeniero Civil, Magíster en Ingeniería Civil, Doctor (c) en Ingeniería. Docente de la Universidad Distrital Francisco José de Caldas. Bogotá, Colombia. Contacto: esanchez@udistrital.edu.co ORCID: https://orcid.org/0000-0001-7370-8745

Estudiante de arquitectura Universidad del Norte. Barranquilla, Colombia. Contacto: gchueco@uninorte.edu.co ORCID: https://orcid. org/0000-0002-9159-8639

Ingeniero Civil, Especialista en Vías. Universidad Distrital Francisco José de caldas. Bogotá, Colombia. Contacto: resquivelr@udistrital.edu.co ORCID: https://orcid.org/0000-0002-6048-7098
} 


\section{INTRODUCCIÓN}

Un experimento se puede definir como una prueba o serie de pruebas en las que se hacen cambios deliberados en las variables de entrada de un proceso o sistema para observar e identificar las razones de los cambios que pudieran observarse en la variable de salida (Montgomery, 2005). Teniendo en cuenta esto, se puede pensar que el diseño de experimentos está relacionado en gran parte con la industria (Aad et al., 2011). Sin embargo, el ámbito de aplicación incluye el análisis de una amplia cantidad de fenómenos, tales como: emisiones generadas por los automotores debido al estado de las vías, la presión lateral ejercida por el hormigón en estado fresco sobre el encofrado (Santilli, Puente, \& Tanco, 2011), o el desempeño de los pavimentos teniendo en cuenta las condiciones de fabricación.

En el campo de aplicación de la ingeniería de pavimentos, el diseño y análisis de experimentos ha sido empleado en diferentes condiciones, por ejemplo para evaluar el efecto del contenido de cal en la clasificación y separación de HMA (Khodaii, Haghshenas, \& Kazemi Tehrani, 2012), para optimizar la confiabilidad de la base granular cuando se estabiliza con cemento (Tan, Zaimoglu, Hinislioglu, \& Altun, 2005), o bien para evaluar la condición del pavimento, la resistencia del pavimento, la incidencia del estado superficial del pavimento sobre el consumo de combustible, las emisiones generadas por los vehículos y el grado de accidentalidad (Cunto \& Saccomanno, 2008; Madanat, Prozzi, \& Han, 2017)(2.

En este artículo se presenta la planeación, desarrollo y análisis de un experimento para analizar los factores que influyen en la resistencia a la compresión (carga máxima kN.) de un material granular compuesto por arcilla y arena. Como se trata de un material que se utiliza en la construcción de parte de la estructura de un pavimento, se utiliza como instrumento de experimentación la norma INVIAS (Instituto Nacional de Vías, Colombia), sección 600 como base técnica y se proponen diferentes combinaciones de rangos para el desarrollo de la fase experimental, con el propósito de conocer cuál sería el comportamiento del material al recibir cargas a compresión.

Metodológicamente se utiliza un diseño de experimento factorial $2^{4}$ que permite conocer cuáles son los factores que tienen efecto significativo sobre la resistencia presentada por el material. El análisis de este experimento permitió proponer el experimento definitivo y estimar el mejor comportamiento del material con relación a la variable respuesta y los factores seleccionados.

Inicialmente, se presenta el experimento de tamizaje que permite identificar los factores que tienen efecto significativo sobre la variable respuesta denominada esistencia a la compresión. Luego se presenta el experimento factorial definitivo. En los dos casos se verifica la adecuación de los modelos propuestos. Posteriormente se hace el análisis de los resultados y se procede a realizar la optimización del diseño del material. Al final son presentadas las conclusiones principales del trabajo.

\section{METODOLOGÍA}

A continuación, se presenta la metodología utilizada para analizar el desempeño del material granular estabilizado con cemento cuando se aplica carga a compresión.

\section{Materiales}

El experimento fue desarrollado en varias etapas. La primera tuvo que ver con la adquisición de cada uno de los materiales a utilizar. La arcilla que se utilizó fue extraída en el relleno sanitario "Los Pocitos" de la empresa Triple A, la arena proviene del municipio de Santo Tomás, situado en el noreste del Departamento del Atlántico, en la región Caribe, ubicado en la ribera del río Magdalena, y parte de su economía gira entorno a la explotación minera (de arena para la construcción), y el cemento Portland Tipo 1, fue producido por Argos (figura 1). 


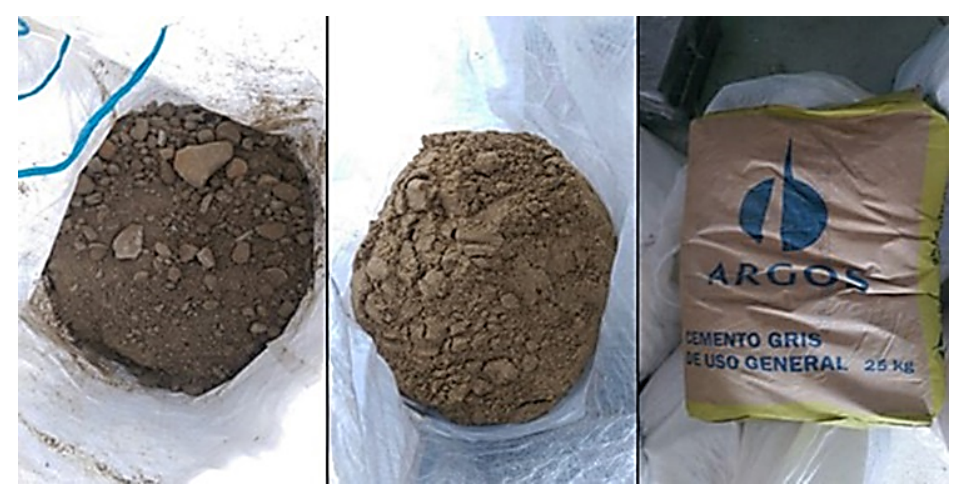

Figura 1. Materiales arcilla, arena y cemento hidráulico Portland

Fuente: elaboración propia.

\section{Experimento de tamizado $2^{4}$}

En general, el diseño $2^{k}$ es utilizado en las etapas iniciales del trabajo experimental ya que proporciona el menor número de corridas con las que pueden estudiarse $k$ factores en un diseño factorial completo (Montgomery, 2005).

El trabajo presentado se ubica en el contexto de análisis del comportamiento de materiales mezclados de tipo granular, cuyos componentes son arciIla y arena, los cuales están presentes en los suelos y pueden constituirse como materiales de sub base sobre los cuales se construyen las vías. Técnicamente el estudio se enmarca en un material granular mezclado cuyos componentes son arcilla y arena, con adición de cemento hidráulico el cual aporta a su estabilización y puede ser utilizado en la estructura de un pavimento, específicamente en la sub base que es la capa que se encuentra entre la base y la sub rasante o terreno natural en un pavimento asfáltico. Debido a que está sometida a menores esfuerzos que la base, su calidad puede ser inferior y generalmente está constituida por materiales locales granulares o marginales.

\section{Diseño}

Como se trata de un material mezclado, para el cual se pueden definir diferentes condiciones de experimentación, se considera que su análisis puede hacerse por la vía de la experimentación. Inicialmente, se pretende lograr identificar los factores más relevantes que inciden en la resistencia presentada por el material cuando se presentan cargas impuestas a compresión.

Normalmente los materiales estabilizados para ser utilizados en sub base contienen adiciones que alteran favorablemente desde el punto de vista del comportamiento ingenieril, algunas propiedades como la textura, la trabajabilidad, la plasticidad y la resistencia (Sabogal, 2007).

Para el diseño, se plantean cuatro factores, siendo todos cuantitativos, tal como se ve en la tabla 1 , para el desarrollo experimental.

De acuerdo con los estudios previos realizados un material granular tipo suelo estabilizado con cemento o simplemente suelo cemento se logra vertiendo sobre él cemento portland, en un porcentaje que puede variar entre un 7 y un $12 \%$ atendiendo al tipo de suelo, y cado uno ofrece comportamientos distintos en cuanto a la resistencia, por tanto, su variación en proporción de los factores es muy relativa a las condiciones del material. El porcentaje de humedad adecuado puede estar entre el 8 y el 16\%, que sería un porcentaje similar al que el suelo posee antes de los trabajos de excavación, aunque puede cambiar dependiendo de las condiciones que se presenten en las obras. Una forma práctica de comprobar si nuestro suelo posee la consistencia deseada, es tomar 
un puñado de este y apretarlo en la mano, así podremos saberlo, si el puñado se cohesiona sin ensuciar la mano y la porción compactada puede partirse en dos entonces tenemos un suelo con la consistencia adecuada.

Tabla 1. Factores y niveles del diseño experimental.

\begin{tabular}{|c|c|c|c|}
\hline Factor & Descripción & Código & Nive \\
\hline \multirow{2}{*}{ A } & \multirow{2}{*}{$\begin{array}{l}\text { Contenido de cemento } \\
\left(\mathrm{Kg} . / \mathrm{m}^{3}\right)\end{array}$} & - & 0 \\
\hline & & + & 150 \\
\hline \multirow{2}{*}{ B } & \multirow{2}{*}{ Humedad (\%) } & - & 9 \\
\hline & & + & 16 \\
\hline \multirow{2}{*}{$\mathrm{C}$} & \multirow{2}{*}{$\begin{array}{l}\text { No. de golpes } \\
\text { de compactación }\end{array}$} & - & 25 \\
\hline & & + & 35 \\
\hline \multirow{2}{*}{$\mathrm{D}$} & \multirow{2}{*}{ Relación (Arcilla/Arena) } & - & 1 \\
\hline & & + & 1,5 \\
\hline
\end{tabular}

Fuente: elaboración propia.

La variable de respuesta del experimento, expresada Kilo newtons (kN.), corresponde a la carga máxima soportada por el material antes de presentar falla, la cual se define como la pérdida de funcional de un material tanto por deformación (fluencia) como por separación de sus partes (fractura). La figura 2, muestra el estado de un especimen cuando es sometido a carga máxima y sufre deformación.

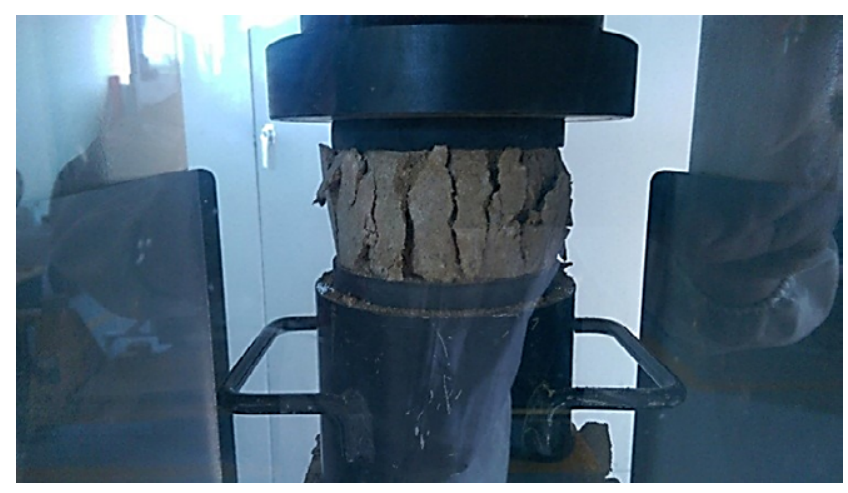

Figura 2. Falla de briqueta

Fuente: elaboración propia.

Tabla 2. Condiciones experimentales del experimento de tamizaje

\begin{tabular}{cccccccccc}
\hline Corrida & A & B & C & D & Etiquetas & A & B & C & D \\
\hline 1 & - & - & - & - & -1 & 0 & 9 & 25 & 1 \\
\hline 2 & + & - & - & - & a & 150 & 9 & 25 & 1 \\
\hline 3 & - & + & - & - & b & 0 & 16 & 25 & 1 \\
\hline 4 & + & + & - & - & ab & 150 & 16 & 25 & 1 \\
\hline 5 & - & - & + & - & c & 0 & 9 & 35 & 1 \\
\hline 6 & + & - & + & - & ac & 150 & 9 & 35 & 1 \\
\hline 7 & - & + & + & - & bc & 0 & 16 & 35 & 1 \\
\hline 8 & + & + & + & - & abc & 150 & 16 & 35 & 1 \\
\hline 9 & - & - & - & + & d & 0 & 9 & 25 & 1,5 \\
\hline 10 & + & - & - & + & ad & 150 & 9 & 25 & 1,5 \\
\hline 11 & - & + & - & + & bd & 0 & 16 & 25 & 1,5 \\
\hline 12 & + & + & - & + & abd & 150 & 16 & 25 & 1,5 \\
\hline 13 & - & - & + & + & cd & 0 & 9 & 35 & 1,5 \\
\hline 14 & + & - & + & + & acd & 150 & 9 & 35 & 1,5 \\
\hline 15 & - & + & + & + & bcd & 0 & 16 & 35 & 1,5 \\
\hline 16 & + & + & + & + & abcd & 150 & 16 & 35 & 1,5 \\
\hline 17 & 0 & 0 & 0 & 0 & & 75 & 12,5 & 30 & 1,25 \\
\hline 18 & 0 & 0 & 0 & 0 & & 75 & 12,5 & 30 & 1,25 \\
\hline 19 & 0 & 0 & 0 & 0 & & 75 & 12,5 & 30 & 1,25 \\
\hline 20 & 0 & 0 & 0 & 0 & & 75 & 12,5 & 30 & 1,25 \\
\hline & & & & & & & & &
\end{tabular}

Fuente: elaboración propia. 
Los factores seleccionados hacen parte de los factores de diseño a tener en cuenta en un material que hará parte importante en la estructura de un pavimento, específicamente en la sub base. Dependiendo de la proporción elegida y de las condiciones de la infraestructura se pueden alcanzar menores o mayores tiempos de vida útil en una carretera. Las corridas del diseño en notación geométrica (Montgomery, 2005) son mostradas en la tabla 2.

Como se trata de un experimento de selección de factores se propuso la realización de una réplica para obtener un total de 16 observaciones acorde con las condiciones experimentales, y una réplica en cada punto central para un total de 20 datos, que se considera una muestra suficiente para esta primera parte del análisis. El experimento fue aleatorizado mediante la generación de números aleatorios, cuyo orden fue seguido durante el proceso de aplicación de cargas estáticas a compresión y falla.

Atendiendo las condiciones experimentales se diseñó cada mezcla, según dosificación de cada material, para luego hacer cada una de las mezclas, las cuales se iban colocando en los moldes para compactación y luego se procedió a compactar, de manera secuencial y simultánea

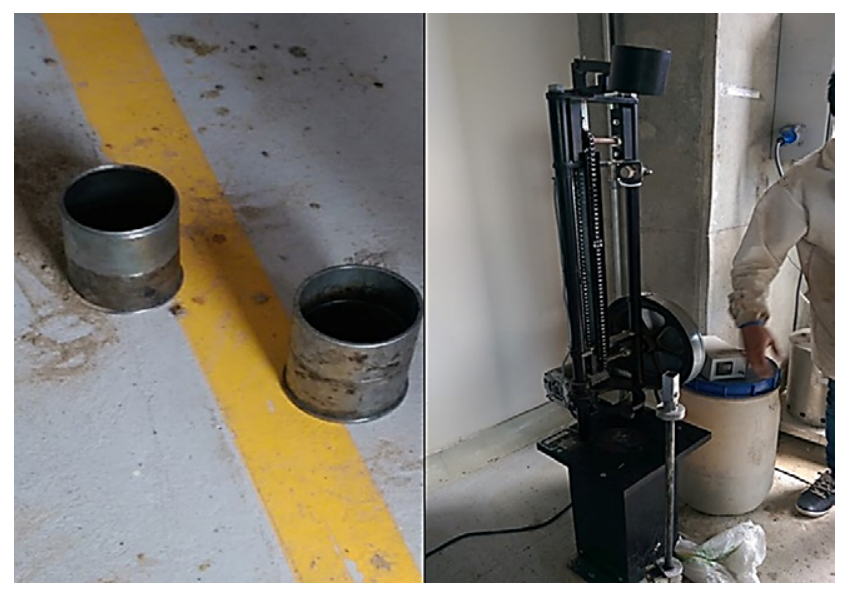

Figura 3. Moldes para fabricación de briquetas y máquina Humboldt para compactación automática Marshall

Fuente: elaboración propia.
La figura 3, muestra los moldes utilizados en la fabricación de las briquetas y el equipo para compactar. Los moldes tienen 4" de diámetro con doble anillo y fondo y la máquina utilizada para compactación es de tipo automático marca Humboldt, tipo 02, modelo H1336, lo cual permite uniformidad en la energía de compactación. Las briquetas elaboradas fueron almacenadas a temperatura ambiente promedio en laboratorio $\left(22^{\circ} \mathrm{C}\right)$, para ser falladas a compresión.

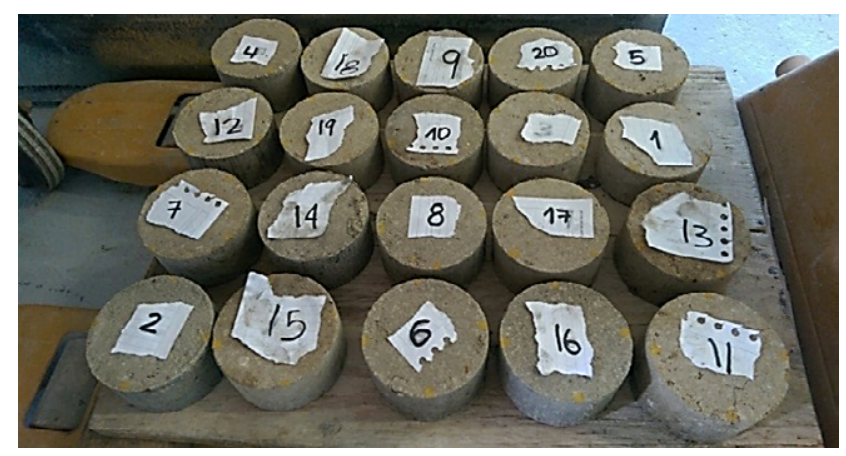

Figura 4. Briquetas fabricadas

Fuente: elaboración propia.

La figura 4, muestra las briquetas elaboradas. La falla se realizó en el orden aleatorio en una máquina de compresión marca Controls modelo 04700/er. La figura 5, muestra la máquina utilizada para extraer las briquetas del molde, y la Figura 6, muestra la máquina utilizada para aplicar la carga a las briquetas.

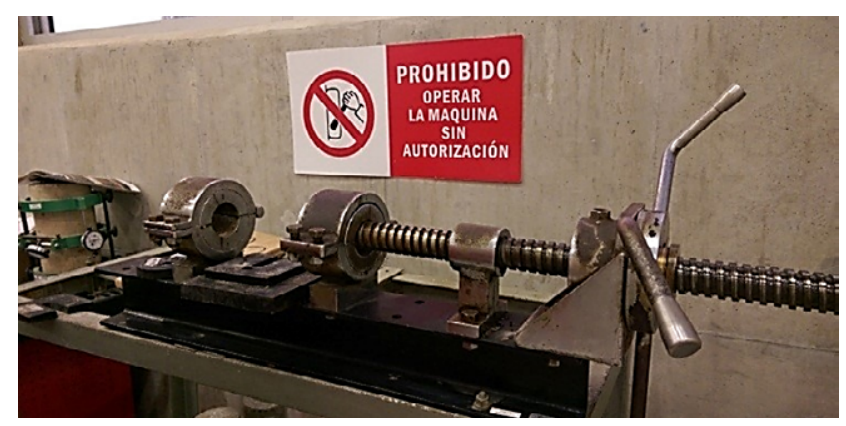

Figura 5. Máquina utilizada para sacar las briquetas del molde

Fuente: elaboración propia. 


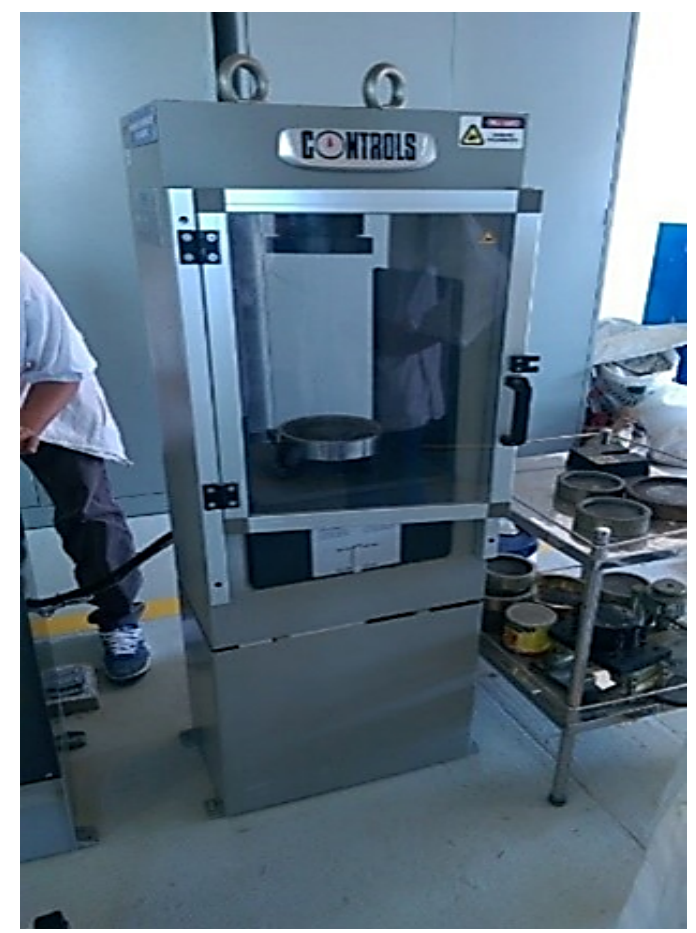

Figura 6. Máquina de compresión marca Controls modelo 04700/er

Fuente: elaboración propia.

Tabla 3. Datos del experimento de tamizado.

\section{RESULTADOS}

La variable de respuesta resistencia a la compresión medida en $\mathrm{kN}$. se ubicó en un rango entre 4 y 17,7 , con un promedio de 8,72 y desviación estándar igual a 3,88.

La tabla 3, muestra los resultados de carga máxima a compresión en cada una de las condiciones experimentales.

La figura 7, muestra una imagen obtenida en la lectura No. 17 para la máxima carga.

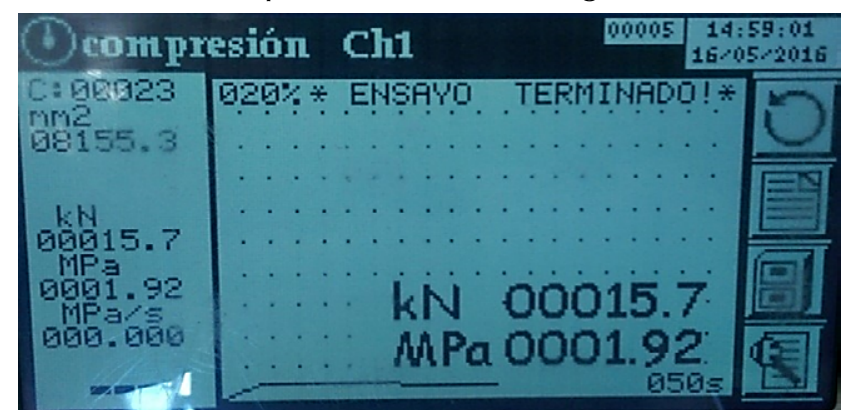

Figura 7. Lectura No. 17 de carga máxima en la máquina de compresión marca Controls modelo 04700/er

Fuente: elaboración propia.

\begin{tabular}{|c|c|c|c|c|}
\hline $\begin{array}{c}\text { Contenido de } \\
\text { cemento }\left(\mathrm{Kg} . / \mathrm{m}^{3}\right)\end{array}$ & $\begin{array}{c}\text { Humedad } \\
(\%)\end{array}$ & $\begin{array}{l}\text { Golpes de compactación } \\
\text { (Unidad) }\end{array}$ & $\begin{array}{l}\text { Relación Arcilla/ } \\
\text { Arena }\end{array}$ & $\begin{array}{l}\text { Resistencia a la compresión } \\
\text { (Carga máxima en kN.) }\end{array}$ \\
\hline 0 & 16 & 25 & 1,5 & 4 \\
\hline 150 & 9 & 25 & 1,5 & 12,2 \\
\hline 150 & 16 & 25 & 1,5 & 10,4 \\
\hline 0 & 9 & 25 & 1 & 6,7 \\
\hline 0 & 9 & 35 & 1,5 & 5,5 \\
\hline 75 & 12,5 & 30 & 1,25 & 8,1 \\
\hline 75 & 12,5 & 30 & 1,25 & 7,7 \\
\hline 75 & 12,5 & 30 & 1,25 & 8,2 \\
\hline 150 & 9 & 35 & 1 & 12,2 \\
\hline 75 & 12,5 & 30 & 1,25 & 9,3 \\
\hline 0 & 9 & 35 & 1 & 4,9 \\
\hline 0 & 16 & 35 & 1 & 4,4 \\
\hline 0 & 16 & 35 & 1,5 & 5,1 \\
\hline 150 & 9 & 35 & 1,5 & 11,1 \\
\hline 0 & 16 & 25 & 1 & 4,7 \\
\hline 150 & 16 & 25 & 1 & 10,8 \\
\hline 150 & 9 & 25 & 1 & 15,7 \\
\hline 150 & 16 & 35 & 1,5 & 17,7 \\
\hline 0 & 9 & 25 & 1,5 & 5 \\
\hline 150 & 16 & 35 & 1 & 10,7 \\
\hline
\end{tabular}

Fuente: elaboración propia. 
La figura 8, muestra el gráfico de probabilidad normal para la resistencia a la compresión, e indica que el factor contenido de cemento tiene efecto significativo, a diferencia de los demás factores (Humedad, golpes de compactación y relación arcilla/arena) que no resultaron significativos.

A su vez, también se puede observar que las interacciones Humedad-Golpes de compactación y Golpes de compactación-Relación arcilla/arena son significativas, mientras que las demás interacciones presentadas no lo son.

Dados los resultados del gráfico de probabilidad en el diseño factorial $2^{k}$, éste se tomará como base para definir los factores significativos para la variable resistencia a la compresión.

Teniendo en cuenta el gráfico de probabilidad normal, se decide que el factor Contenido de cemento es significativo, mientras que las interacciones no lo son, dado que los factores generadores de estas no presentan significancia. La Tabla 4, muestra el análisis de varianza incluida la prueba de falta de ajuste para el análisis de puntos centrales se presenta a continuación.

La tabla 4 muestra que la prueba de falta de ajuste es mayor que 0,05 no es significativa, por tanto, el modelo parece ser adecuado para los datos observados al nivel de confianza del 95,0\%., y la curvatura que se presenta debido a los puntos centrales no es significativa, lo que puede deducir un modelo lineal.

La tabla 5, muestra el estadístico R-Cuadrado, que indica que el modelo, así ajustado, explica $80,1472 \%$ de la variabilidad de la Resistencia a la compresión. El estadístico R-cuadrado ajustado, que es más adecuado para comparar modelos con diferente número de variables independientes, es 79,0442\%. El error estándar del estimado muestra que la desviación estándar de los residuos es 1,81313. El error medio absoluto (MAE) de 1,15225 es el valor promedio de los residuos.

Gráfico de Probabilidad Normal para Resistencia a la compresión

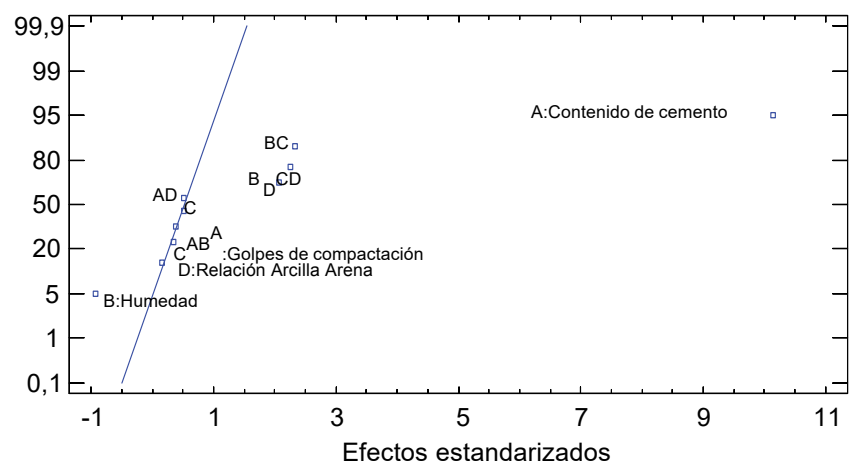

Figura 8. Gráfico de probabilidad Normal para la variable respuesta (Resistencia a la compresión)

Fuente: elaboración propia.

Tabla 4. Análisis de varianza factores significativos y falta de ajuste

\begin{tabular}{lccccc}
\hline \multicolumn{1}{c}{ Fuente } & $\begin{array}{c}\text { Suma de } \\
\text { cuadrados }\end{array}$ & $\begin{array}{c}\text { Grados de } \\
\text { libertad }\end{array}$ & $\begin{array}{c}\text { Cuadrado } \\
\text { medio }\end{array}$ & F-razón & P-valor \\
\hline A: Contenido de cemento & 228,766 & 1 & 228,766 & 69,59 & 0,0000 \\
\hline Falta de ajuste & 0,780125 & 1 & 0,780125 & 0,24 & 0,6324 \\
\hline Error puro & 558,862 & 17 & 328,743 & & \\
\hline Total (corr.) & 285,432 & 19 & & & \\
\hline
\end{tabular}

Fuente: elaboración propia. 
El estadístico de Durbin-Watson (DW) prueba los residuos para determinar si hay alguna correlación significativa basada en el orden en que se presentan los datos en el archivo. Puesto que el valor-P es mayor que 5,0 \%, no hay indicación de auto correlación serial en los residuos con un nivel de significancia del 5,0\%.

Tabla 5. Estadísticos del modelo

\begin{tabular}{ll}
\hline $\mathrm{R}^{2}(\%)$ & 80,1472 \\
\hline $\mathrm{R}^{2}$ (ajustada por grados de libertad $(\%)$ & 79,0442 \\
\hline Error estándar del estadístico & 181,313 \\
\hline Error absoluto medio & 115,225 \\
\hline Estadístico Durbin-Watson & $1,54523(\mathrm{P}=0,1494)$ \\
\hline Auto correlación residual de Lag 1 & 0,190982 \\
\hline
\end{tabular}

Fuente: elaboración propia.

La ecuación (1) presenta el modelo ajustado, donde los valores de las variables están especificados en sus unidades originales.

Resistencia a la compresión $=4.93875+$ 0.0504167(Contenido de cemento)

\section{Verificación de supuestos}

\section{Supuesto de normalidad}

Para verificar si los residuos pueden modelarse adecuadamente con una distribución normal, se utiliza la prueba de Shapiro-Wilk que está basada en la comparación de los cuartiles de la distribución normal ajustada a los datos.

La tabla 6, muestra los resultados de la prueba Shapiro - Wilk. Debido a que el valor-P es mayor a 0,05 , no se puede rechazar la idea de que los residuos provienen de una distribución normal con 95\% de confianza.

La figura 9, muestra el gráfico elaborado, que evidencia que no hay sospecha de no cumplir el supuesto de normalidad.

Tabla 6. Prueba de normalidad para los residuos del experimento de tamizado

\begin{tabular}{ccc}
\hline Prueba & Estadístico & Valor-P \\
\hline Estadístico W de Shapiro-Wilk & 0,952713 & 0,420723 \\
\hline
\end{tabular}

Fuente: elaboración propia.

\section{Supuesto de independencia}

La auto correlación residual de Lag 1 es 0,175891 para los residuos de la variable de respuesta Resistencia a la compresión, y muestra que se cumple el supuesto de independencia. La figura 10, muestra el gráfico de secuencias cronológicas para los residuos.

La figura 10 no muestra una tendencia de comportamiento de los residuos del experimento de tamizado, lo que indica que los residuos cumplen el supuesto de independencia.

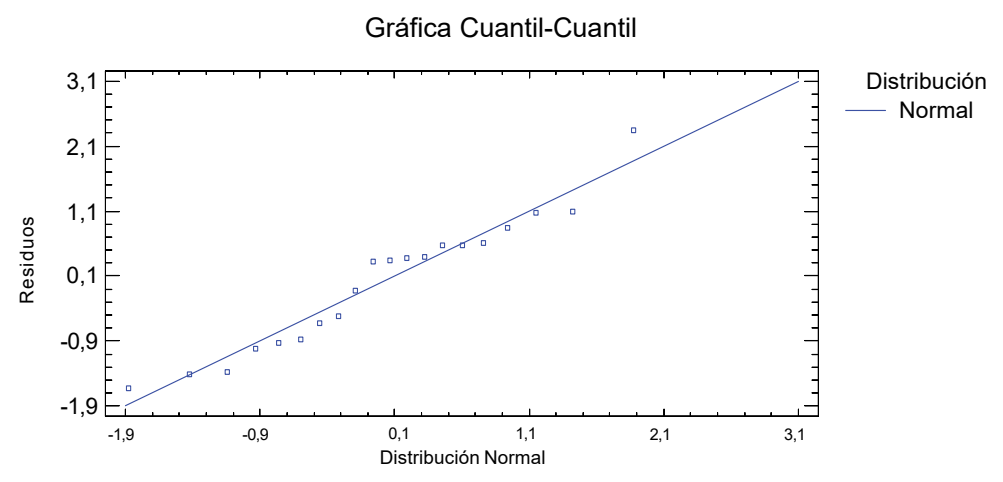

Figura 9. Gráfico de distribución Normal para los residuos del experimento de tamizado

Fuente: elaboración propia. 


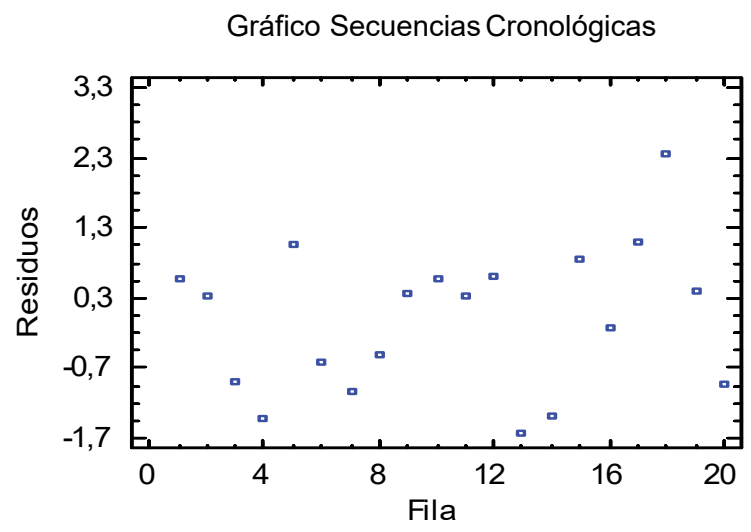

Figura 10. Gráfico de secuencias cronológicas para los residuos del experimento de tamizado

Fuente: elaboración propia.

\section{Supuesto de homocedasticidad}

Para evaluar el cumplimiento del supuesto de homocedasticidad de tomo el gráfico de residuos, que se muestra en la figura 11, para la resistencia a la compresión y se determinó la razón del intervalo mayor entre el intervalo menor que fue de 2,33. Por ser menor a 5 , se considera que los residuos cumplen con el supuesto de homocedasticidad.

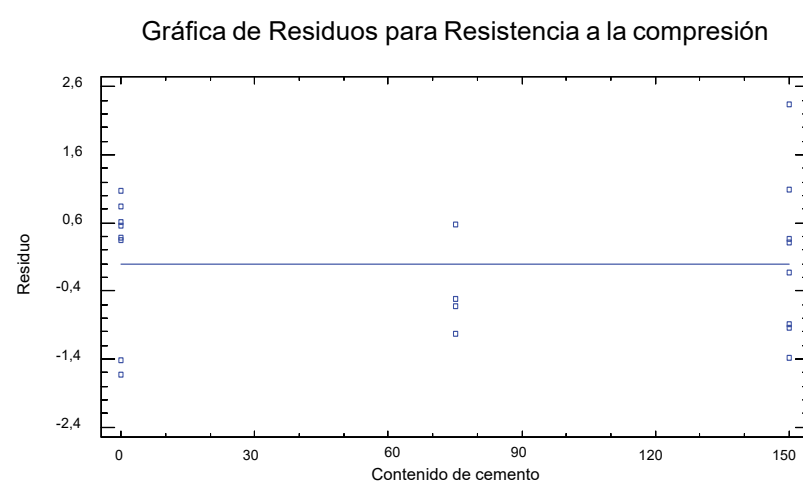

Figura 11. Gráfico de residuos para la Resistencia a la compresión en el experimento de tamizado

Fuente: elaboración propia.

\section{Optimización}

Teniendo en cuenta que la mayor carga y resistencia que se presenta en las condiciones experimentales planteadas se encuentra cuando las condiciones de cada uno de los factores son altas, y que el factor Contenido de cemento es significativo, se plantea recorrer un camino buscando un óptimo fuera de la región experimental manteniendo los factores humedad, golpes de compactación y relación Arcilla/Arena en los niveles altos como lo muestra la tabla 7 .

Tabla 7. Optimización de respuesta

\begin{tabular}{lccc}
\hline \multicolumn{1}{c}{ Factor } & Bajo & Alto & Óptimo \\
\hline Contenido de cemento & 0,0 & 150,0 & 150,0 \\
\hline Humedad & 9,0 & 16,0 & 16,0 \\
\hline Golpes de compactación & 25,0 & 35,0 & 35,0 \\
\hline Relación Arcilla Arena & 1,0 & 1,5 & 1,5 \\
\hline
\end{tabular}

Fuente: elaboración propia.

La tabla 7, muestra la combinación de los niveles de los factores, la cual maximiza resistencia a la compresión sobre la región indicada. Para realizar la optimización manteniendo los factores no significativos en alto, el factor Contenido de cemento se incrementará en tres valores fuera de la región de experimentación. Los valores serán 175, 200 y $225 \mathrm{~kg}$. por $\mathrm{m} 3$ de material granular mezclado y se elaborarán dos briquetas en cada condición experimental. La tabla 8, muestra los resultados de carga máxima $(\mathrm{kN})$ para la optimización. 
Tabla 8. Datos experimento para optimización

\begin{tabular}{ccccc}
\hline $\begin{array}{c}\text { Contenido de } \\
\text { cemento } \mathbf{( K g} . \mathbf{m} 3)\end{array}$ & $\begin{array}{c}\text { Humedad } \\
\mathbf{( \% )}\end{array}$ & $\begin{array}{c}\text { Golpes de compactación } \\
\text { (Unidad) }\end{array}$ & $\begin{array}{c}\text { Relación } \\
\text { Arcilla/Arena }\end{array}$ & $\begin{array}{c}\text { Resistencia a la compresión } \\
\text { (Carga máxima kN.) }\end{array}$ \\
\hline 225 & 16 & 35 & 1,5 & 14,1 \\
\hline 200 & 16 & 35 & 1,5 & 16,3 \\
\hline 225 & 16 & 35 & 1,5 & 14,5 \\
\hline 200 & 16 & 35 & 1,5 & 14,9 \\
\hline 175 & 16 & 35 & 1,5 & 11,9 \\
\hline 175 & 16 & 35 & 1,5 & 13,2 \\
\hline
\end{tabular}

Fuente: elaboración propia.

Al definir el diseño, se desarrolló la fase experimental en laboratorio, bajo las mismas condiciones de la fase experimental inicial del diseño $2^{\mathrm{k}}$. Se ajustaron las dosificaciones a fin de cumplir con las proporciones y el diseño que mantiene las condiciones (alto) de los factores humedad, número de golpes y relación arcilla/arena.

Con la información que incluyó la variable respuesta se realizó un análisis de varianza de un solo factor, que se presenta en la tabla 9. Es importante mencionar que el análisis de varianza (ANOVA), no muestra que el factor cantidad de cemento sea significativo.

Teniendo en cuenta lo anterior, se esperaría que con la tendencia de ascenso de la variable respuesta hacia la frontera de la región experimental, en el camino fuera de ella se aumentara el valor de la variable contenido de cemento genere incremento en la variable respuesta resistencia a la compresión.

Se desarrolla entonces un diseño de un solo factor (Contenido de cemento) con tres niveles (175, 200 y $225 \mathrm{Kg}$. /m3) y dos réplicas en cada nivel.

La figura 12, muestra el comportamiento de la variable Resistencia a la compresión en cada uno de los niveles de diseño. En este caso se nota que la resistencia cae en la condición de $175 \mathrm{Kg} . / \mathrm{m} 3$, para luego aumentar en la condición de $200 \mathrm{Kg}$. $/ \mathrm{m} 3$ y subir nuevamente en la condición de $225 \mathrm{Kg} . / \mathrm{m} 3$.

El valor máximo alcanzado en la condición de 200 Kg. / m3 no supera el valor máximo alcanzado en la frontera de la región experimental planteada inicialmente $150 \mathrm{Kg}$. / m3, lo que inicialmente permite suponer que el valor máximo de la variable respuesta Resistencia a la compresión se encuentra en esta condición.

\section{Verificación de supuestos}

\section{Supuesto de normalidad}

Para verificar si los residuos pueden modelarse adecuadamente con una distribución normal, se utiliza la prueba de Shapiro-Wilk que está basada en la comparación de los cuartiles de la distribución normal ajustada a los datos.

La tabla 10, muestra los resultados para determinar si los residuos pueden modelarse adecuadamente con una distribución normal.

Tabla 9. Análisis de varianza de un solo factor (Contenido de cemento)

\begin{tabular}{lccccc}
\hline \multicolumn{1}{c}{ Fuente } & $\begin{array}{c}\text { Suma de } \\
\text { cuadrados }\end{array}$ & $\begin{array}{c}\text { Grados de } \\
\text { libertad }\end{array}$ & Cuadrado medio & Razón-F & valor-P \\
\hline Entre grupos & 9,37 & 2 & 4,685 & 7,38 & 0,0694 \\
\hline Intra grupos & 1,905 & 3 & 0,635 & & \\
\hline Total (Corr.) & 11,275 & 5 & & & \\
\hline
\end{tabular}

Fuente: elaboración propia. 


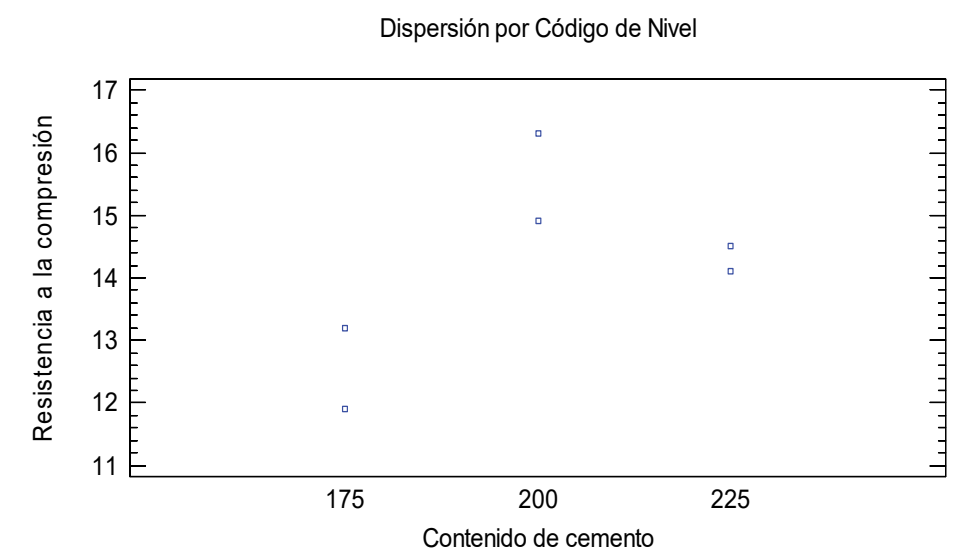

Figura 12. Gráfico de dispersión por nivel del experimento factorial

Fuente: elaboración propia.

Debido a que el valor-P más pequeño de las pruebas realizadas es mayor a 0,05 , no se puede rechazar la idea que los residuos provienen de una distribución normal con 95\% de confianza. La figura 13, muestra el gráfico de cuartil -cuartil.

Tabla 10. Prueba de normalidad para los residuos del experimento factorial

\section{Supuesto de independencia}

La auto correlación residual de Lag 1 es 0,056 para los residuos de la variable de respuesta Resistencia a la compresión, y muestra que se cumple el supuesto de independencia. La figura 14, muestra el gráfico de secuencias cronológicas para los residuos.

\begin{tabular}{ccc}
\hline Prueba & Estadístico & Valor-P \\
\hline Estadístico W de Shapiro-Wilk & 0,990394 & 0,988049 \\
\hline
\end{tabular}

Fuente: elaboración propia.

Gráfica Cuantil-Cuantil

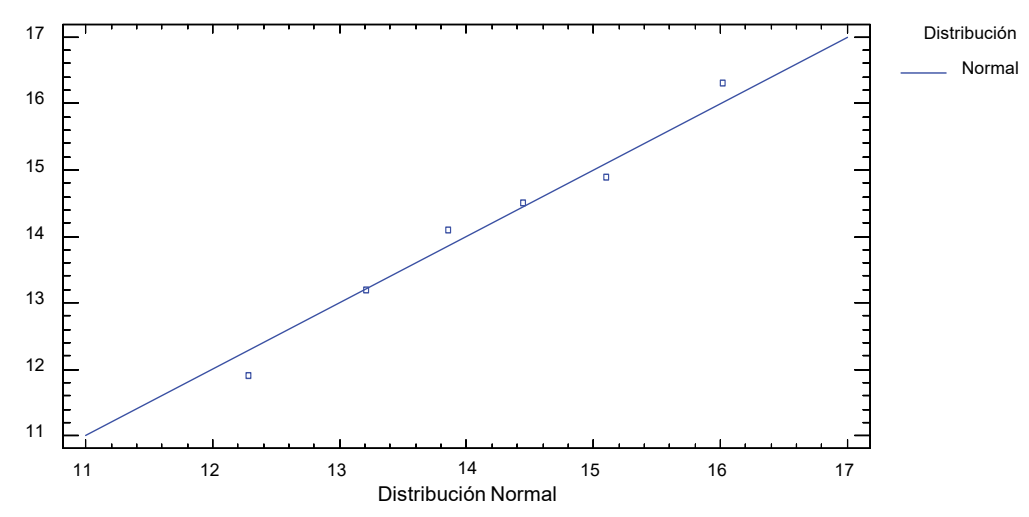

Figura 13. Gráfico de distribución Normal para los residuos del experimento factorial

Fuente: elaboración propia. 


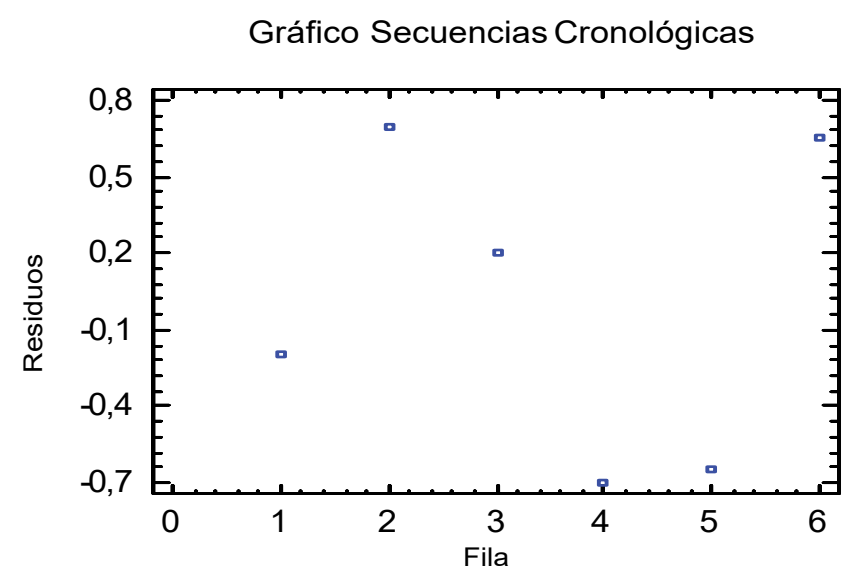

Figura 14. Gráfico de secuencias cronológicas para los residuos del experimento factorial

Fuente: elaboración propia.

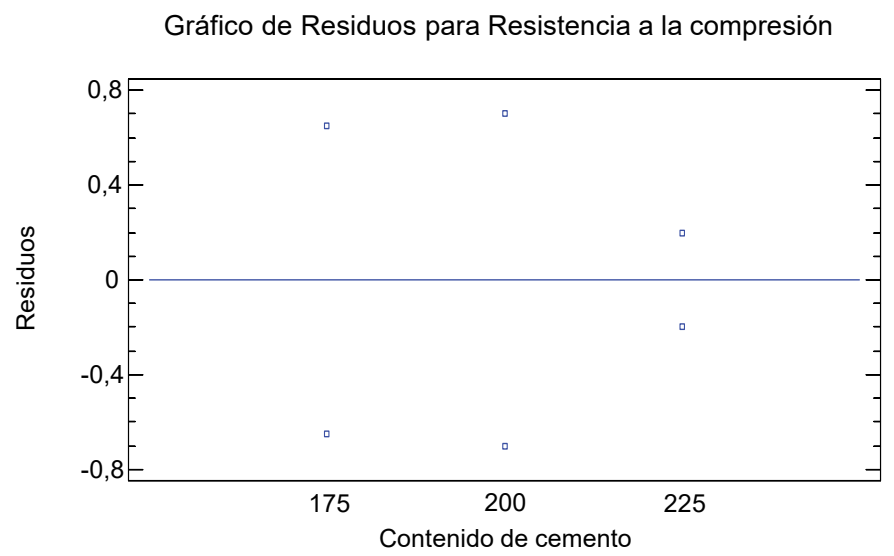

Figura 15. Gráfico de residuos para la Resistencia a la compresión en el experimento factorial

Fuente: elaboración propia.

\section{Supuesto de homocedasticidad}

Para evaluar el cumplimiento del supuesto de homocedasticidad de tomo el gráfico de residuos para la resistencia a la compresión y se determinó la razón del intervalo mayor entre el intervalo menor que fue de 3,5. Por ser menor a 5, se considera que los residuos cumplen con el supuesto de homocedasticidad. La figura 15, muestra el gráfico de residuos para la Resistencia a la compresión.

\section{CONCLUSIONES}

Estudiada en forma experimental la resistencia que presenta un material compuesto por arcilla, arena, cemento y agua en distintas proporciones. Un experimento de tamizaje $2^{4}$ con una réplica y cuatro (4) puntos centrales encontró que la humedad, el número de golpes de compactación y la relación arcilla/arena, no produce un efecto significativo 
sobre la resistencia a la compresión (carga máxima medida en kN.), mientras que el contenido de cemento si produce un efecto significativo sobre la variable respuesta.

Mediante un experimento factorial desarrollado fuera de la región experimental, pero atendiendo el comportamiento del factor contenido de cemento, se identificó un cambio en el comportamiento de la variable respuesta sin llegar a ser mejor que la encontrada en la frontera de la región experimental.

Se verificó el cumplimiento de los supuestos de normalidad, independencia y homocedasticidad para dar validez a los análisis hechos durante este trabajo.

Finalmente, el trabajo corrobora los rangos sugeridos para contenido de cemento para capas de base estabilizadas en la clasificación A3, A4, A5, A6 y A7, utilizadas para carreteras por la AASHTO, los cuales coinciden con los valores encontrados, de tal forma que la relación de $150 \mathrm{Kg} . / \mathrm{m} 3$, puede considerarse como adecuada al momento de diseñar este tipo de materiales que sirven de soporte a varios elementos en los proyectos de construcción, tanto en la cimentación de las edificaciones, como en la estructura que se conforma como soporte de los pavimentos.

\section{AGRADECIMIENTOS}

Agradecimientos por el apoyo a la Ingeniera Andrea Castro, jefe de laboratorio de materiales del Departamento de Ingeniería Civil de la Universidad del Norte y a su auxiliar de laboratorio Julio Coba.

\section{REFERENCIAS}

Aad, G., Abbott, B., Abdallah, J., Abdelalim, A. A., Abdesselam, A., Abdinov, O., ... Zutshi, V. (2011). Measurement of inclusive jet and dijet cross sections in proton-proton collisions at $7 \mathrm{TeV}$ centre-of-mass energy with the ATLAS detector. European Physical Journal C, 71(2), 1-59.

https://doi.org/10.22323/1.120.0101

Cunto, F., \& Saccomanno, F. F. (2008). Calibration and validation of simulated vehicle safety performance at signalized intersections. Accident Analysis and Prevention, 40(3), 1171-1179.

https://doi.org/10.1016/j.aap.2008.01.003

Khodaii, A., Haghshenas, H. F., \& Kazemi Tehrani, H. (2012). Effect of grading and lime content on HMA stripping using statistical methodology. Construction and Building Materials, 34, 131-135.

https://doi.org/10.1016/j.conbuildmat.2012.02.025

Madanat, S. M., Prozzi, J. A., \& Han, M. (2017). Effect of Performance Model Accuracy on Optimal Pavement Design. UC Berkeley.

https://doi.org/10.1038/nnano.2017.155

Montgomery, D. C. (2005). Diseño y análisis de experimentos. (L. Wiley, Ed.).

Santilli, A., Puente, I., \& Tanco, M. (2011). A factorial design study to determine the significant parameters of fresh concrete lateral pressure and initial rate of pressure decay. Construction and Building Materials, 25(4), 1946-1955.

https://doi.org/10.1016/j.conbuildmat.2010.11.061

Tan, O., Zaimoglu, A. S., Hinislioglu, S., \& Altun, S. (2005). Taguchi approach for optimization of the bleeding on cement-based grouts. Tunnelling and Underground Space Technology, 20(2), 167-173.

https://doi.org/10.1016/j.tust.2004.08.004

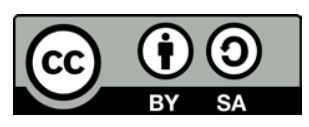

Tecnura • p-ISSN: 0123-921X • e-ISSN: 2248-7638 • Vol. 23 No. $60 \bullet$ Abril - Junio de 2019・pp. 59-71 